\title{
ON CHERN CLASSES OF REPRESENTATIONS OF FINITE GROUPS
}

\author{
BY J. KNOPFMACHER
}

Communicated by E. Dyer, December 21, 1964

Let $R(G)$ denote the complex representation ring of a finite group $G$. Any complex representation $\rho$ of $G$ has invariants $c_{n}(\rho) \in H^{2 n}(G ; Z)$, the Chern classes of $\rho$ (Atiyah [1]).

If $H$ is a subgroup of $G$, there is the induced representation homomorphism

$$
i_{!}: R(H) \rightarrow R(G)
$$

(cf. [8], say). Atiyah [1] posed the problem of relating the Chern classes of $i_{i} \lambda$ with those of $\lambda$, for any representation $\lambda$ of $H$. The purpose of this note is to announce the proof of a conjecture of J. F. Adams which gives some information in this direction; the main idea of the proof was suggested to me by Professor Adams, and is believed to emanate essentially from Professor Atiyah. I would like to thank Professor Adams sincerely for his help, and to acknowledge the helpfulness of Professor Atiyah and Professor M. G. Barratt.

The result to be proved involves the transfer homomorphism

$$
i_{1}: H^{*}(H ; Z) \rightarrow H^{*}(G ; Z)
$$

(cf. [6], [8]), and certain linear maps

$$
\mathrm{Ch}_{k}: R(L) \rightarrow H^{2 k}(L ; \boldsymbol{Z})
$$

defined, for any finite group $L$, in terms of the Chern classes as follows:

Let $Q^{k}\left(\sigma_{1}, \cdots, \sigma_{n}\right)$ be the polynomial defined by expressing the symmetric polynomial $x_{1}^{k}+\cdots+x_{n}^{k}$ in indeterminates $x_{1}, \cdots, x_{n}$ in terms of the elementary symmetric polynomials $\sigma_{i}\left(x_{1}, \cdots, x_{n}\right)$. If $\rho: L \rightarrow U(n)$ is a representation of $L$ of degree $n$, then

$$
\mathrm{Ch}_{k}(\rho)=Q^{k}\left(c_{1}(\rho), \cdots, c_{n}(\rho)\right) \in H^{2 k}(L ; \boldsymbol{Z}) .
$$

Theorem 1. Given any positive integer $k$, there exists an integer $N_{k}$ with the following property:

If $H$ is an arbitrary subgroup of an arbitrary finite group $G$, then the following diagram of homomorphisms commutes: 


$$
\begin{array}{lc}
R(H) \stackrel{i_{!}}{\longrightarrow} & R(G) \\
N_{k} \mathrm{Ch}_{k} \downarrow & \downarrow N_{k} \mathrm{Ch}_{k} \\
H^{2 k}(H ; \boldsymbol{Z}) \stackrel{i_{!}}{\longrightarrow} & H^{2 k}(G ; \boldsymbol{Z}) .
\end{array}
$$

If $N_{k}$ denotes the least positive integer with this property, and $G^{\prime}$ is a group of order prime to $N_{k}$, it follows that

$$
\mathrm{Ch}_{k} i_{!}=i_{!} \mathrm{Ch}_{k}
$$

for all monomorphisms $i: H \rightarrow G^{\prime}$.

The case $k=0$ of this theorem is trivial. The case $k=1$ follows from a previously unpublished lemma of J. F. Adams below, while the case $k>1$ is dealt with, along lines suggested by Professor Adams, by means of a certain "Riemann-Roch type" lemma (Lemma 3).

Lemma 2 (J. F. Adams). If $\lambda \in R(H)$, then

$$
c_{1}\left(i_{!} \lambda\right)=i_{!} c_{1}(\lambda)+(\operatorname{deg} \lambda) c_{1}\left(i_{!} 1\right),
$$

where 1 is the trivial representation of $H$.

This lemma is proved by exploiting a very explicit algebraic description of the first Chern class (cf. [1]), and standard explicit algebraic descriptions of the maps $i_{!}$(cf. [8], say). The proof shows that $c_{1}\left(i_{1} 1\right)$ always has order dividing 2 . Further, the example of $\{1\} \subset \boldsymbol{Z}_{2}$ shows that 2 is the least positive integer $N_{1}$ such that $N_{1}\left(\mathrm{Ch}_{1} i_{1}-i_{!} \mathrm{Ch}_{1}\right)$ $\equiv 0$.

The general case of the theorem is deduced from the following lemma:

LeMmA 3. Let $f: X \rightarrow Y$ be a covering map of compact almost-complex manifolds. Then the following diagram commutes:

$$
\begin{array}{cc}
K^{*} \mathrm{c}(X) \stackrel{f_{!}}{\longrightarrow} & K^{*} \mathrm{C}(Y) \\
M_{k} \mathrm{Ch}_{k} \downarrow & \downarrow M_{k} \mathrm{Ch}_{k}, \\
H^{*}(X ; Z) \stackrel{f_{!}}{\longrightarrow} H^{*}(Y ; Z)
\end{array}
$$

where $M_{k}=\prod_{r=1}^{k}(n+r) ! / r !, 2 n=\operatorname{dim}_{R} X, Y$, and the maps $f_{!}$are those given by using Thom isomorphisms defined by normal bundles to $X$ and $Y$.

OUtLINE OF Proof. First suppose that $f: X \rightarrow Y$ is an arbitrary map of almost-complex manifolds. Let $\phi_{H}, \phi_{K}$ denote Thom isomorphisms 
in integral cohomology and in $K$-theory defined by normal bundles to a given almost-complex manifold $W$. Write

$$
B_{k}(W)=\phi_{H}^{-1} \mathrm{Ch}_{k} \phi_{K}(1) .
$$

By methods similar to some used in [5] one obtains the formula:

$$
\sum_{r=0}^{k}\left(\begin{array}{l}
k \\
r
\end{array}\right)\left[B_{r}(Y) \cdot \mathrm{Ch}_{k-r} f_{1} x-f_{1}\left(B_{r}(X) \cdot \mathrm{Ch}_{k-r} x\right)\right]=0\left[x \in K_{C}^{*}(X)\right] .
$$

In the case that $f$ is a finite covering, $B_{r}(X)=f^{*} B_{r}(Y)$. Further, if $2 n=\operatorname{dim}_{R} X, Y$, the Bott results on $K_{C}\left(S^{2 n}\right)$ imply that $B_{n}(Y)=n !$. Hence, in this case, the formula reduces to the equation

$$
\begin{aligned}
& \frac{k !}{(k-n) !}\left(\mathrm{Ch}_{k-n} f_{1} x-f_{1} \mathrm{Ch}_{k-n} x\right) \\
& \quad=-\sum_{r=n+1}^{k}\left(\begin{array}{l}
k \\
r
\end{array}\right) B_{r}(Y)\left[\mathrm{Ch}_{k-r} f_{1} x-f_{l} \mathrm{Ch}_{k-r} x\right]\left[x \in K_{C}^{*}(X)\right] .
\end{aligned}
$$

The required result now follows by induction.

The following lemma is an immediate consequence of a result of J.-P. Serre (quoted in [2]).

Lemma 4. Let $H$ be a subgroup of a finite group G. For any integer $n>2$, there exists a covering map $p: X_{H} \rightarrow X_{G}$ of projective complex algebraic manifolds both of (real) dimension $2(n+1)$ and such that $X_{H}, X_{G}$ have the same homotopy n-type as products of Eilenberg-MacLane spaces $K(\boldsymbol{Z}, 2) \times K(H, 1), K(\boldsymbol{Z}, 2) \times K(G, 1)$, respectively.

The required theorem is now proved in dimension $2 k$ by considering a covering map of this type when $n=2 k$, and applying Lemma 3. (A step of this kind was suggested in a letter by Professor Atiyah.) In that case it remains to be shown that the maps $p_{1}$ coincide with the algebraically-defined transfer maps. This is accomplished with the aid of results which appear in [1], [7], [3] and [5]; these results reduce the problem finally to that of comparing the $K$-theory transfer map with that defined by Grothendieck (cf. [4]) in terms of sheaves. (This is done in a final lemma.)

\section{REFERENCES}

1. M. F. Atiyah, Characters and cohomology of finite groups, Inst. Hautes Etudes Sci. Publ. Math. No. 9, Paris, 1961.

2. M. F. Atiyah and F. Hirzebruch, Analytic cycles on complex manifolds, Topology 1 (1961), 25-45. 
3. - The Riemann-Roch theorem for analytic embeddings, Topology 1 (1962), 151-166.

4. A. Borel and J.-P. Serre, Le théorème de Riemann-Roch (d'après Grothendieck), Bull. Soc. Math. France 86 (1958), 97-136.

5. E. Dyer, Relations between cohomology theories, Aarhus Colloquium on Algebraic Topology, 1962; pp. 89-93.

6. B. Eckmann, Cohomology of groups and transfer, Ann. of Math. (2) 58 (1953), 481-493. 35.

7. - On complexes with operators, Proc. Nat. Acad. Sci. U.S.A. 39 (1953),

8. M. Hall, Jr., Theory of groups, Macmillan, New York, 1959.

The University, Manchester, England and

University of WitwatersRand, Johannesburg, South Africa

\section{ISOMORPHIC COMPLEXES}

\section{BY JACK SEGAL}

\section{Communicated by M. L. Curtis, December 28, 1964}

In this paper we show that if $K$ and $L$ are $n$-complexes, then $K$ and $L$ are isomorphic iff the 1-sections of the first derived complexes of $K$ and $L$ are isomorphic. This provides a low-dimensional method for establishing the isomorphism (homeomorphism) of complexes (polyhedra).

Throughout, $s_{p}$ will denote a (rectilinear) $p$-simplex with vertices $a^{0}, a^{1}, \cdots, a^{p} ; K$ will denote a (finite geometric) complex with $n$ section $K^{n}$ and first derived complex $K^{\prime}$. The closed star of a vertex $a$ of $K$, st $(a)$, is the set of simplexes of $K$ having $a$ as a face and all their faces. For more details see [2].

Definition 1. An $n$-complex $K$ is full provided, for any subcomplex $L$ of $K$ which is isomorphic to $s_{p}^{1}, 2 \leqq p \leqq n, L^{0}$ spans a $p$-simplex of $K$.

Theorem 1. Suppose $K$ and $L$ are full $n$-complexes. Then $K$ and $L$ are isomorphic iff $K^{1}$ and $L^{1}$ are isomorphic.

Proof. We need only consider the case when $K^{1}$ and $L^{1}$ are isomorphic. Let $v: K^{1} \rightarrow L^{1}$ be an admissible vertex transformation of $K^{1}$ onto $L^{1}$ with an admissible inverse. Then $a^{0}, a^{1}$ span a 1 -simplex of $K$ iff $v\left(a^{0}\right), v\left(a^{1}\right)$ span a 1-simplex of $L$. Furthermore, for any $p$, $2 \leqq p \leqq n$, if $a^{0}, a^{1}, \cdots, a^{p}$ span a $p$-simplex $s_{p}$ of $K$, then $v\left[s_{p}^{1}\right]$ is isomorphic to $s_{p}^{1}$. So, using the fullness of $L$, we get that $\left(v\left[s_{p}^{1}\right]\right)^{0}$ 